Supporting Information

\title{
Bio-inspired Design of Reinforced Gradient Hydrogels with Rapid Water-Triggered Shape Memory Performance
}

Quanyi Yin, Shuhua Tu, Min Chen* and Limin Wu

Department of Materials Science and State Key Laboratory of Molecular Engineering of Polymers, Advanced Coatings Research Center of Ministry of Education of China,

Fudan University, Shanghai 200433, China

Email: chenmin@fudan.edu.cn 


\section{Materials and methods}

Acrylamide (AAm), N, N'-methylenebis(acrylamide) (MBAA), N, N, N', N'-tetramethylethylenediamine (TEMED), ammonium persulfate (APS) and crystal violet $(\mathrm{CV})$ were purchased from Aladin and used without further purification. Hyperbranched polyethoxysiloxane (PEOS) (1700 g/moL) was synthesized according to the method published elsewhere. ${ }^{1}$ Toluene, ethanol (EtOH), ethyl ether were provided by Sinopharm Chemical Reagent Co., Ltd. Deionized water $\left(\mathrm{H}_{2} \mathrm{O}\right)$ was used throughout the experiments.

\section{Preparation of self-heating driven gradient hydrogels}

$4.69 \mathrm{~g}$ AAm was dissolved into $10 \mathrm{~mL}$ deionized water. After that, MBAA (10 mg), TEMED (20 $\mu \mathrm{L})$ and APS (10 mg) were added. The mixture solution was added drop by drop into the toluene solution of PEOS $(0.5 \mathrm{~g}, 0.75 \mathrm{~g}$ or $1 \mathrm{~g}$ PEOS dissolved in $2 \mathrm{~mL}$ toluene) in ice bath. The weight ratio of PEOS to AAm are $11 \%, 16 \%$ and $22 \%$, respectively. The mixture was gently shaken for $2 \mathrm{~min}$, leading to an oil in water emulsion. Then the mixture solution were injected into desired molds and stored at room temperature overnight for complete polymerization. After the reactions, the hydrogels were peeled from the mold and incubated in $100 \mathrm{~mL}$ of ethyl ether for $12 \mathrm{~h}$ to remove the unreacted monomer and the toluene. The resulted samples were dried under vacuum at R.T. to remove the ethyl ether and then were swelled into water. In order to investigate the influence of PEOS on the properties of hydrogels, several controlled pure PAAm hydrogels were synthesized by using the similar formulation.

\section{Swollen tests of the gradient hydrogels}


Swelling tests were carried out by immersing dried hydrogels in deionized water for a certain time and measuring changes in their mass. The swollen ratio (SR) was defined as $\mathrm{SR}=(\mathrm{Ws}-\mathrm{Wd}) / \mathrm{Wd} * 100 \%$, where $\mathrm{Ws}$ and $\mathrm{Wd}$ represent the weight of hydrogels after being swollen in deionized water with time changing and weight of hydrogels before swelling, respectively. The equilibrium swelling degree (ESD) is defined as, $\mathrm{ESD}=(\mathrm{We}-\mathrm{Wd}) / \mathrm{Wd}$, where We means the weight of samples in swollen equilibrium state.

\section{Cationic dye adsorption}

Cationic dye adsorption experiments were performed by a batch process at room temperature. Dye solutions (20 mg/L) were prepared by dissolving CV in water. The prepared gradient hydrogels with defined weight (after drying) was immersed in 100 $\mathrm{mL}$ of $20 \mathrm{mg} / \mathrm{L} \mathrm{CV}$ dye solution at $25{ }^{\circ} \mathrm{C}$. A small amount of the dye solution was withdrawn from the adsorption system at regular intervals of time for the analysis of the dye concentrations determined by UV-vis spectroscopy using standard calibration curve. Measurements were conducted at $\lambda_{\max }$ of CV (590 nm). Adsorption capacity $\left(q_{t}\right)$ is obtained as the following equations:

$$
q_{t}=\frac{C_{0}-C_{t}}{m} \times V
$$

Where $q_{t}$ is adsorption capacity $(\mathrm{mg} / \mathrm{g})$ at time $t, C_{0}$ and $C_{t}$ are initial and bulk dye concentration at the time $t(\mathrm{mg} / \mathrm{L})$, respectively, $V$ is volume of the solution $(\mathrm{L})$, and $m$ is the mass of the adsorbent $(\mathrm{g})$.

\section{Calculation of shape fixity ratio and shape recovery ratio}


A bend-recover test was used to investigate the water-induced shape memory performance as described elsewhere, ${ }^{2}$ and at least three specimens were tested at R.T. The original samples ( $25 \mathrm{~mm}$ length, $5 \mathrm{~mm}$ width, and $2.5 \mathrm{~mm}$ thickness) were bent in half $\left(180^{\circ}\right)$ in EtOH under external stress for different duration and the temporary angle $\left(\theta_{l}\right)$ after remove from EtOH was obtained. Then the samples were exposed to water, and a digital camera was used to record the whole recovering process. The residual angle was defined as $\theta_{2}$. The shape fixity ratio $\left(R_{f}\right)$ and shape recovery ratio $\left(R_{r}\right)$ of the water-induced shape memory effect were evaluated according to the following formulas:

$R_{r}=\frac{\left(\theta_{1}-\theta_{2}\right)}{\theta_{1}} \times 100 \%, \quad R_{f}=\frac{\theta_{1}}{180} \times 100 \%$

\section{Characterization method}

Optical microscopic observation of emulsions was conducted on a DYS-327C optical microscope. Attenuated total reflection Fourier transform infrared (FT-IR) spectra were recorded using a Nicolet iS10 apparatus with a resolution of $4 \mathrm{~cm}^{-1}$ and a 32 scans accumulation within a range of $500-4000 \mathrm{~cm}^{-1}$. X-ray photoelectron spectroscopy (XPS) was performed with a VG ESCA-LAB220i-XL spectrometer to detect the surface chemical composition of dried hydrogels. The UV-visible spectra of the dye solution were recorded using a UVS-2100 SCINCO spectrophotometer. The absorption spectra of the dilute dye solution in water were recorded in the wavelength range of 300-1000 nm using a $3 \mathrm{~mL}$ silica cell with a path length of 10 $\mathrm{mm}$. The hydrogel structures were characterized by a scanning electron microscope (SEM, SEM Ultra 55, Zeiss, Germany) and computed tomography (CT, source voltage: 
$45 \mathrm{kV}$, image pixel size: $8.70 \mu \mathrm{m}$, filter: Al $0.2 \mathrm{~mm}$ ). The elemental mapping and energy dispersive X-ray (EDX) spectra were performed using a Zeiss Ultra 55 FE-SEM. For the compression tests, the cylindrical samples with a diameter and height of $25 \mathrm{~mm}$ and $5.5 \mathrm{~mm}$, respectively, were placed between the self-leveling plates and compressed at a rate of $10 \mathrm{~mm} / \mathrm{min}$. For tensile test, hydrogels were evaluated by uniaxial tensile tests at $25{ }^{\circ} \mathrm{C}$ using an Instron 5566 universal tensile tester. The samples with a surface dimension of $5 \times 2 \mathrm{~mm}^{2}$ and a thickness of $2.5 \mathrm{~mm}$ were employed. The tension to break was applied with a rate of $60 \mathrm{~mm} / \mathrm{min}$. Rheological measurements were performed using a HAAKE MARS III rhometer with a $25 \mathrm{~mm}$ diameter parallel-plate geometry measuring system. The storage modulus $\left(\mathrm{G}^{\prime}\right)$ and loss modulus $\left(\mathrm{G}^{\prime \prime}\right)$ were recorded from amplitude sweep of hydrogels at a constant frequency of $1 \mathrm{~Hz}$ at a shear stress from $10^{-2}$ to $10^{3} \mathrm{~Pa}$.

(a)

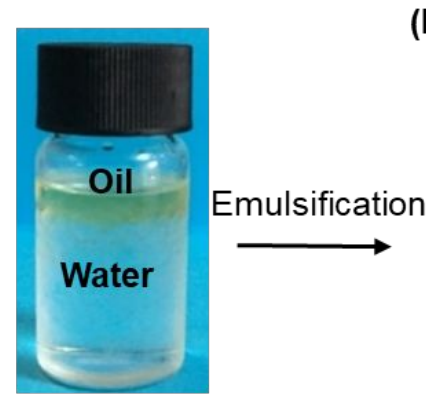

(b)

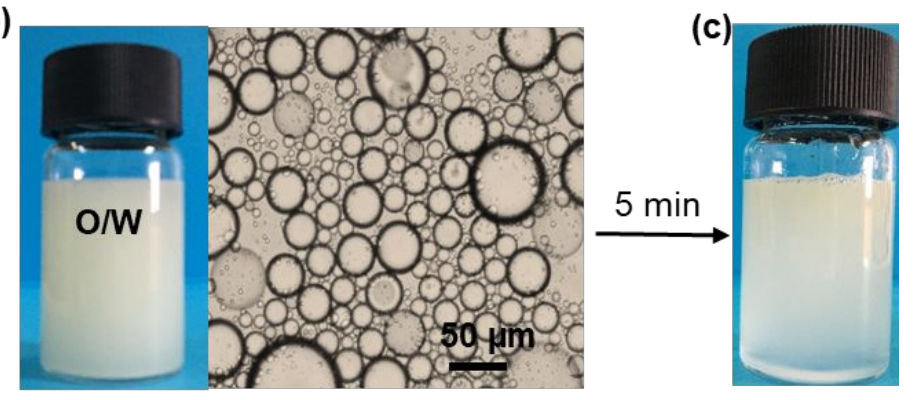

Oil phase: PEOS/Toluene Water phase: AAm/MBAA/APS/TEMED/ $\mathrm{H}_{2} \mathrm{O}$

Figure S1. The photograph of the blend solution of oil and water phase before (a) and after (b) emulsification with the corresponding optical microscopy image of the emulsion; the emulsion after $5 \min (\mathrm{c})$. 

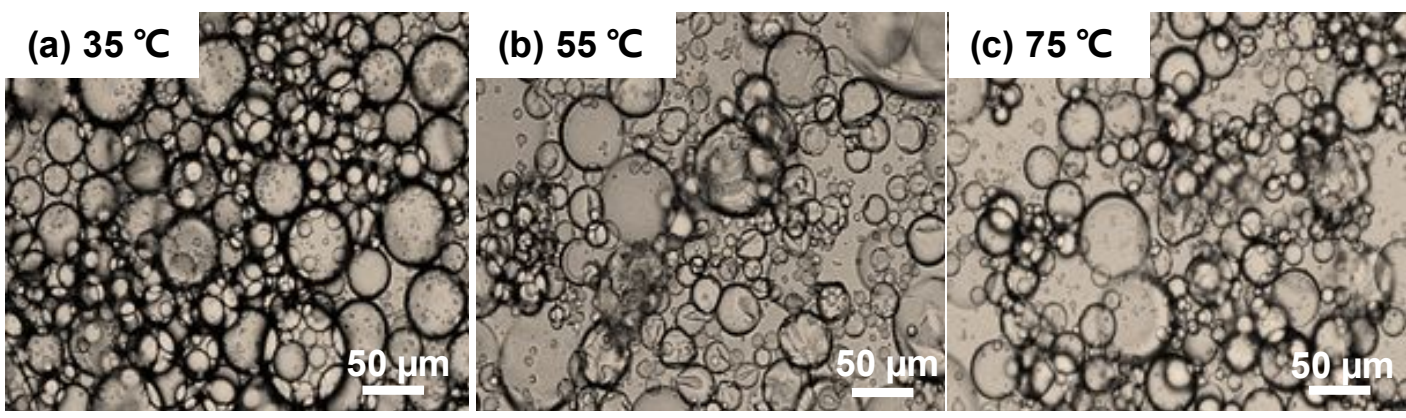

Figure S2. Optical images of the emulsion at $35^{\circ} \mathrm{C}(\mathrm{a}), 55^{\circ} \mathrm{C}(\mathrm{b})$ and $75^{\circ} \mathrm{C}$ (c).
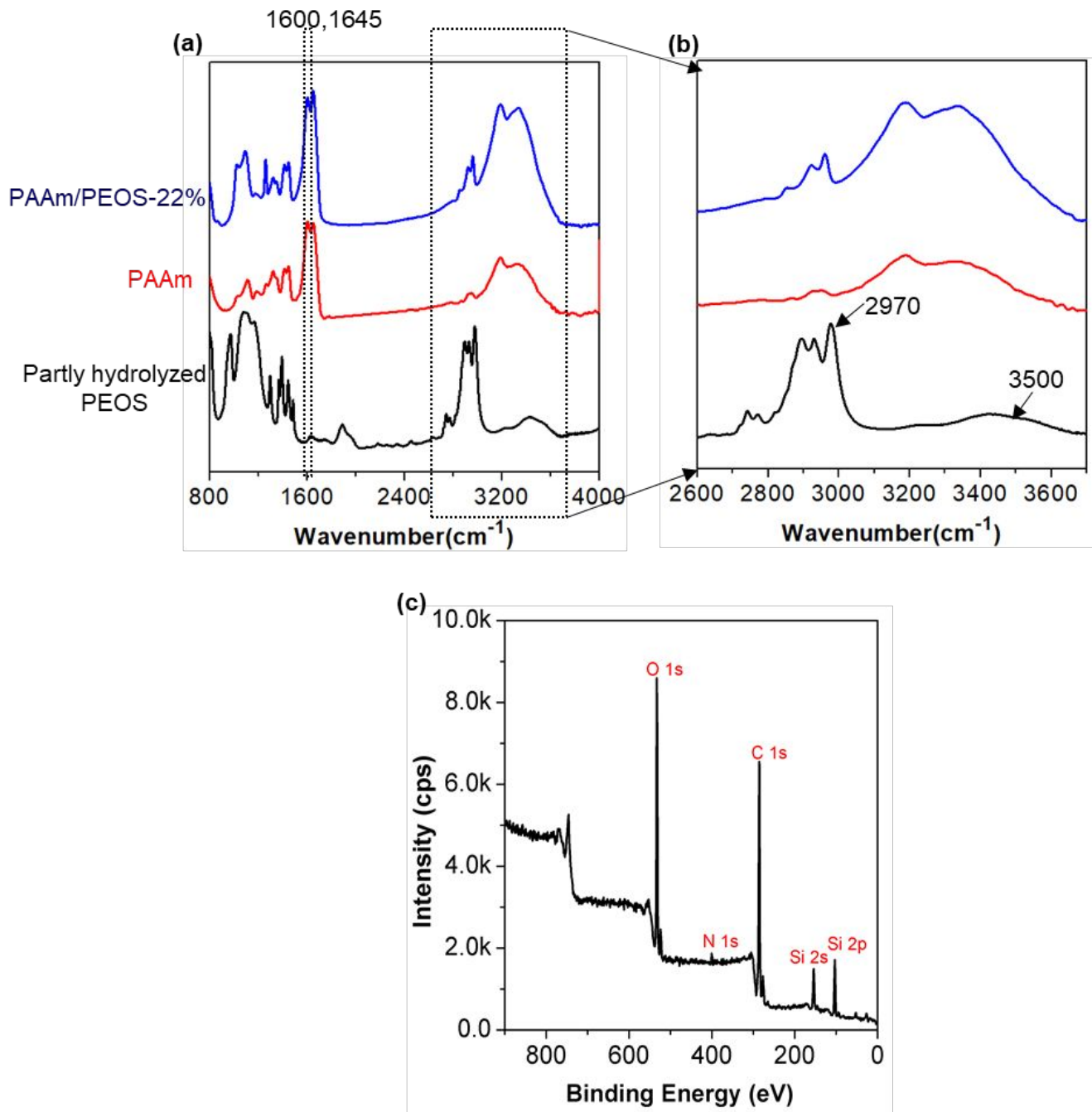

Figure S3. FT-IR spectra of PEOS, PAAm and the gradient hydrogel of PAAm/PEOS-22\% (a and b); (c) XPS spectrum of top surface of PAAm/PEOS-22\%. 

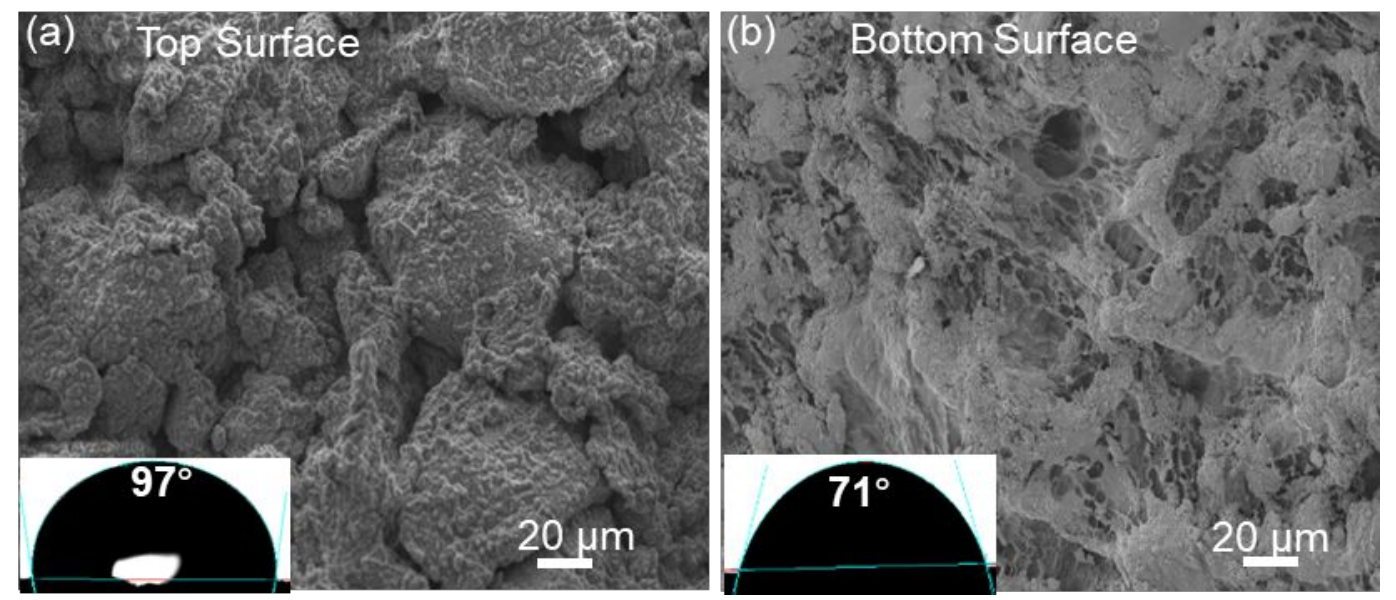

Figure S4. The SEM images of the top (a) and bottom surface (b) of the dried gradient PAAm/PEOS-22\% hydrogel with the corresponding water contact angle image.
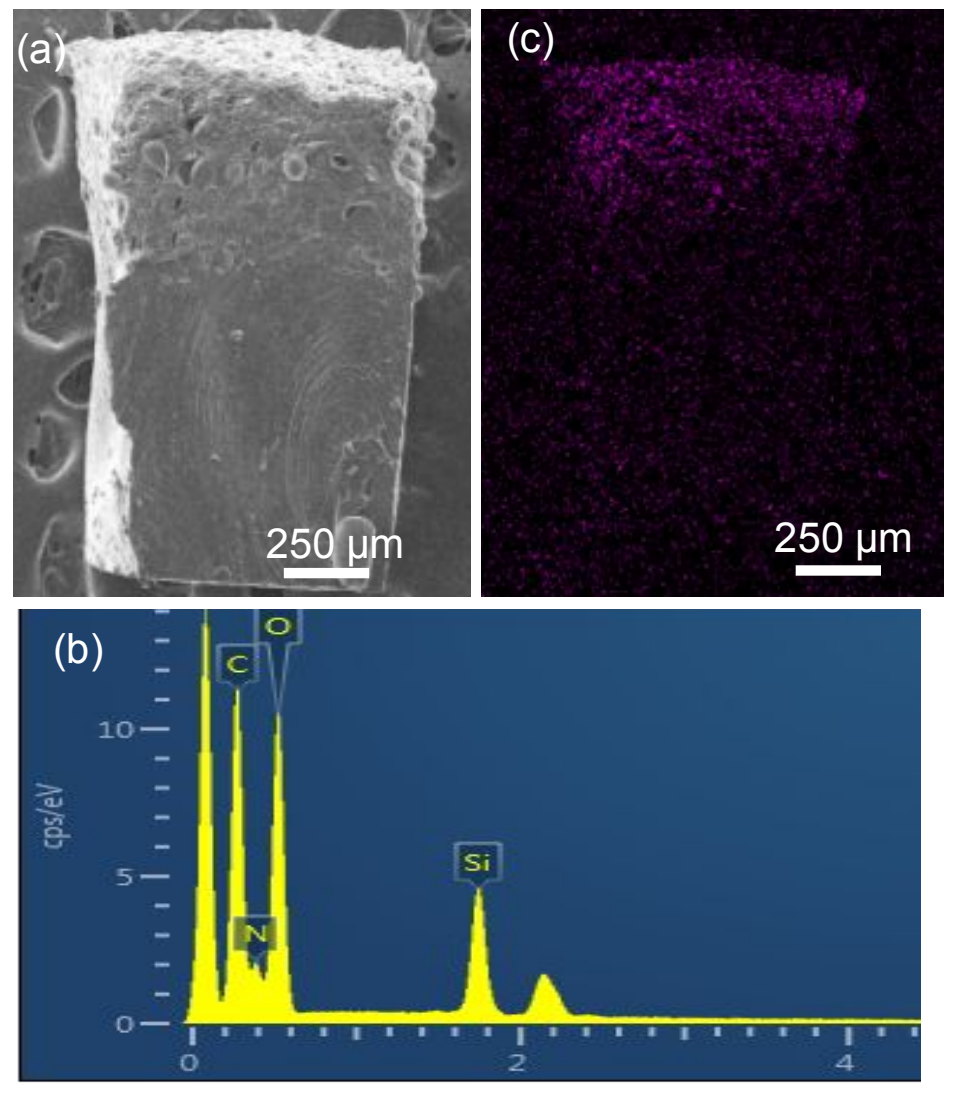

Figure S5. The SEM cross-section of the as-prepared gradient PAAm/PEOS-22\% hydrogel (a) with the corresponding EDX spectra (b) and the silicon element mapping 
image (c).
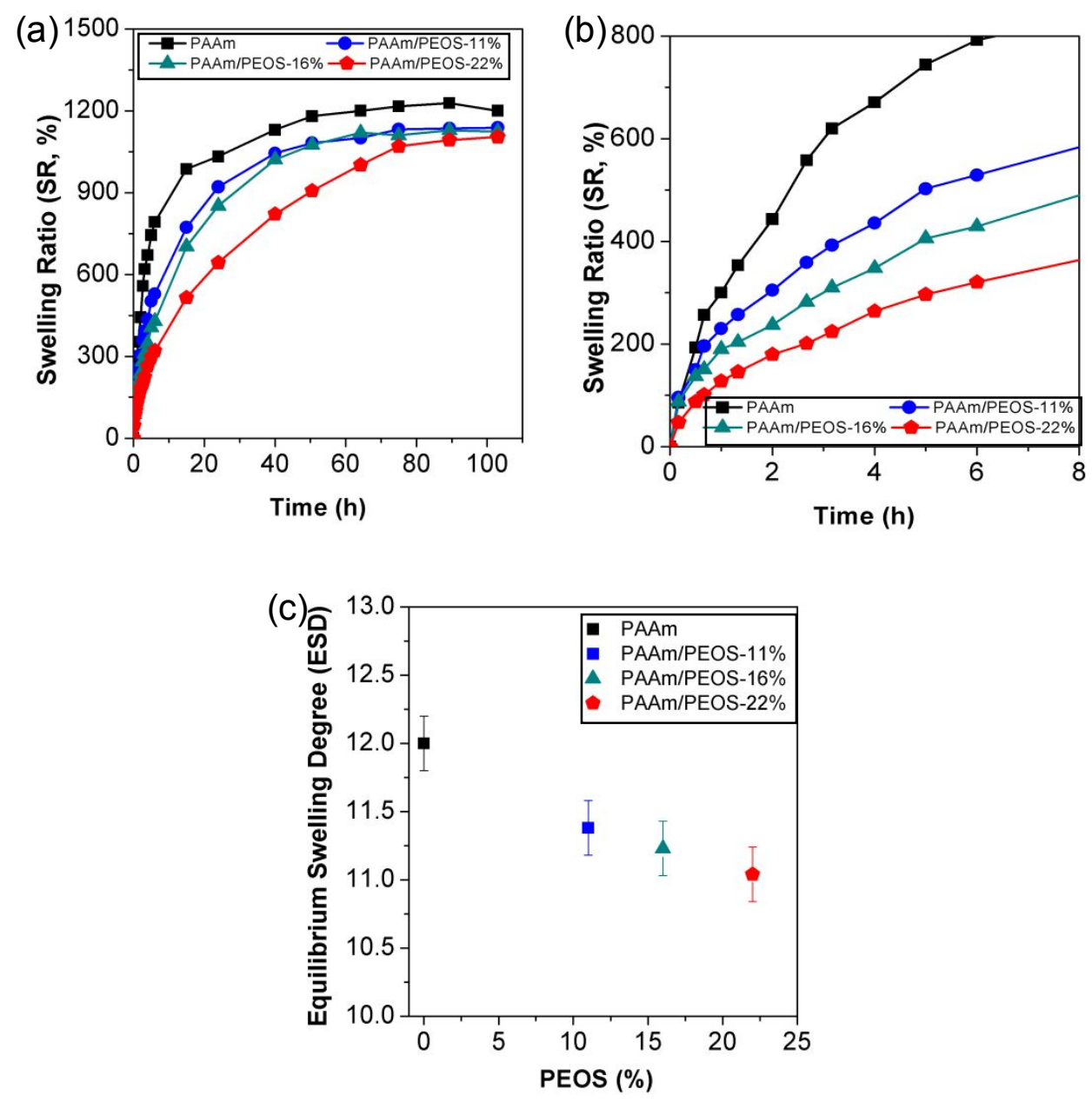

Figure S6. Swelling ratio (SR) tests $(a, b)$ and equilibrium swelling degree (ESD) (c) of the hydrogels with various PEOS contents.

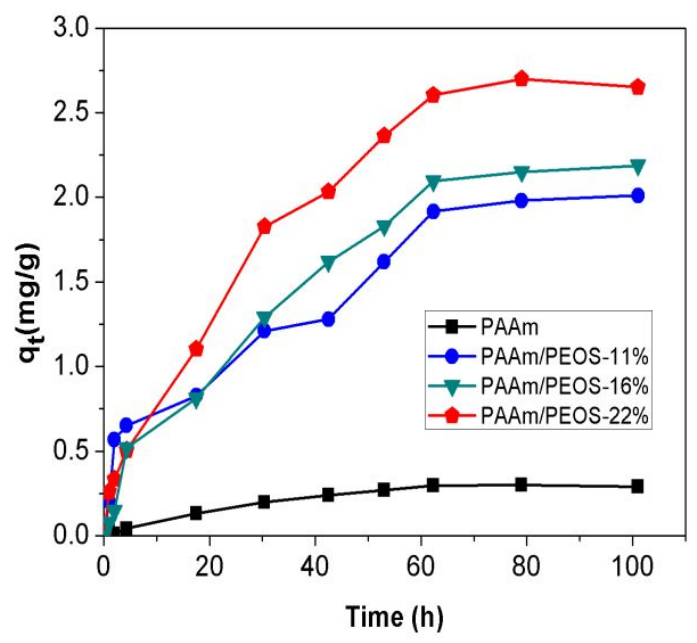


Figure S7. Time profiles of crystal violet $(\mathrm{CV})$ dye adsorption onto PAAm/PEOS hydrogels with different PEOS contents.

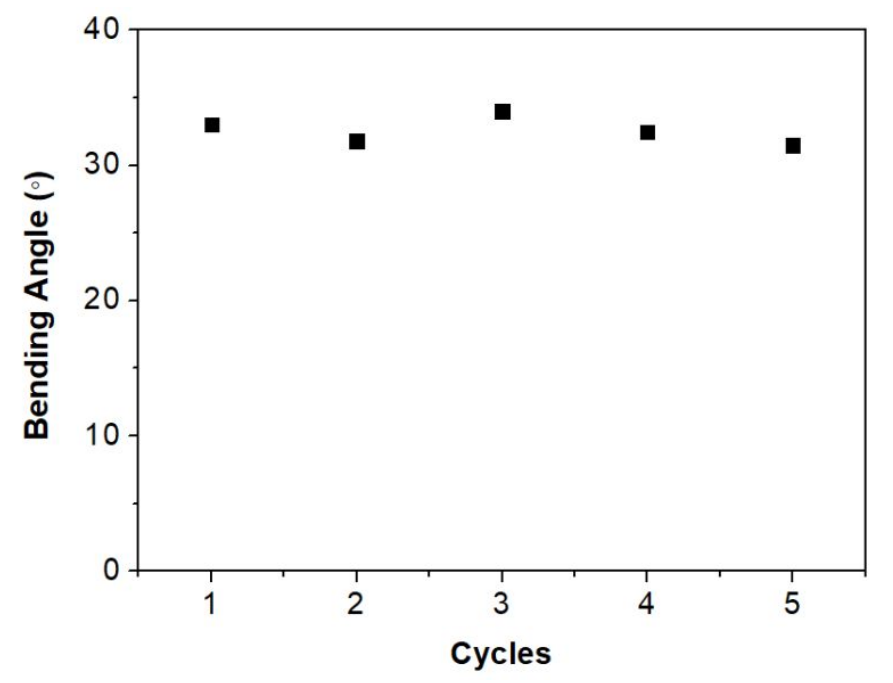

Figure S8. The bending angle changes as a function of performing cycles.

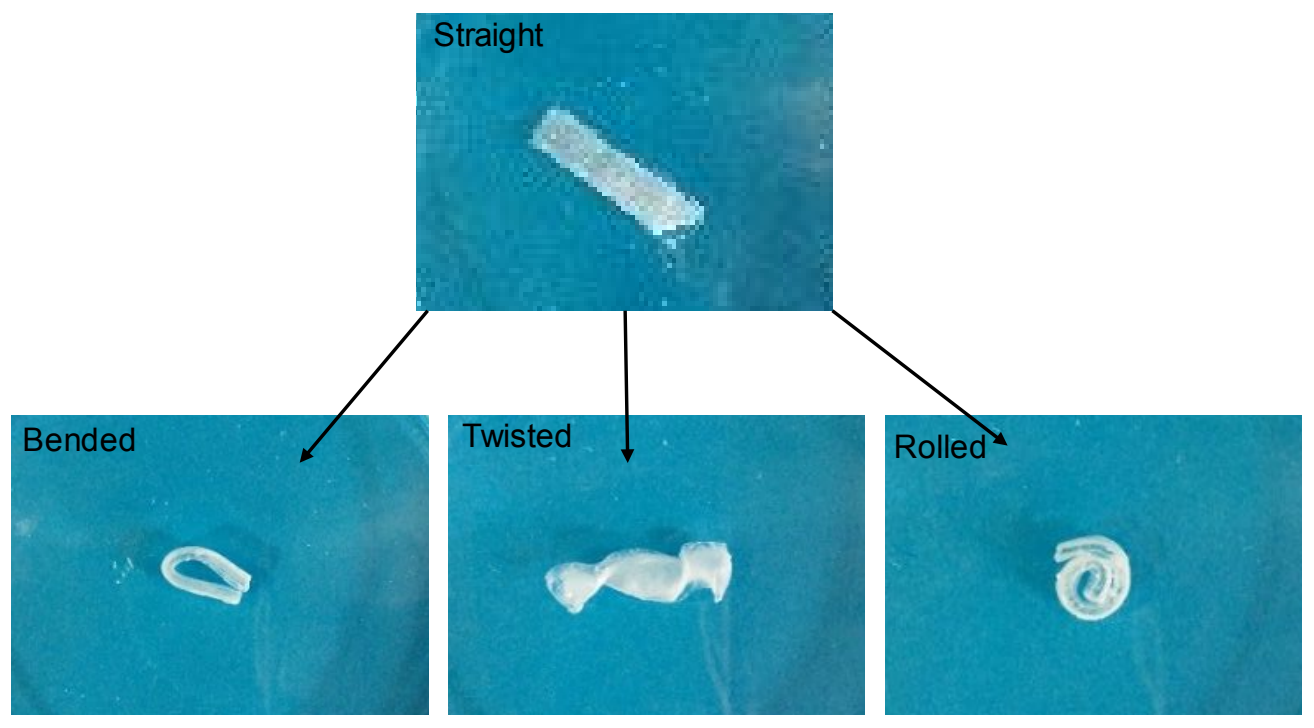

Figure S9. The bending, twisting and rolling of straight PAAm/PEOS-22\% hydrogel.

\section{Reference}

(1) Zhu, X.; Jaumann, M.; Peter, K.; Möller, M.; Melian, C.; Adams-Buda, A.; Demco, D. E.; Blümich, B. One-Pot Synthesis of Hyperbranched Polyethoxysiloxanes. Macromolecules 2006, 39 (5), 1701-1708. 
(2) Qi, X.; Yao, X.; Deng, S.; Zhou, T.; Fu, Q. Water-Induced Shape Memory Effect of Graphene Oxide Reinforced Polyvinyl Alcohol Nanocomposites. $J$. Mater. Chem. A 2014, 2 (7), 2240-2249. 